\title{
Belle II virtual reality projects
}

\author{
Michael Bender ${ }^{1, *}$, Thomas $\mathrm{Kuhr}^{1, * *}$, and Leo Piilonen ${ }^{2, * *}$ \\ ${ }^{1}$ Ludwig-Maximilians-Universität München, Excellence Cluster Universe, Boltzmannstr. 2, 85748 \\ Garching, Germany \\ ${ }^{2}$ Virginia Polytechnic Institute and State University, Blacksburg, Virginia 24061, USA
}

\begin{abstract}
The Belle II experiment, based in Japan, is designed for the precise measurement of B- and charm-meson as well as $\tau$-lepton decays and is intended to play an important role in the search for physics beyond the Standard Model. To visualize the collected data, amongst other things, virtual reality (VR) applications are used within the collaboration. In addition to the already existing VR application which runs on a head-mounted display (HMD), an implementation for the cave automatic virtual environment (CAVE) has been created, where the CAVE is an immersive VR environment, in which projectors are directed to up to six walls of a room-sized cube. These VR applications allow for the inspection of the Belle II detector itself, as well as the illustration of GEANT4 simulated (and data) events of the electron-positron collisions occurring at the SuperKEKB collider. The VR implementations are not only limited to the use within the Belle II collaboration, but are a helpful tool in education and outreach activities.
\end{abstract}

\section{Introduction}

The Belle II detector, shown in figure 1, together with the associated SuperKEKB accelerator, belong to a particle physics experiment at the KEK research facility in Tsukuba, Japan [1]. Belle II has been specifically designed to identify particles created in electron-positron collisions and to measure their properties with high precision. The main goals of Belle II are the precise determination of fundamental physical constants and the search for physics beyond the Standard Model. The detector is located at the interaction point of the SuperKEKB accelerator, where electrons and positrons collide at a center of mass energy of $10.56 \mathrm{GeV}$. With a design luminosity of $8 \cdot 10^{35} \mathrm{~cm}^{-2} \mathrm{~s}^{-1}$, SuperKEKB is planned to break the world record in instantaneous luminosity and will produce an unprecedented amount of

\footnotetext{
*e-mail: Michael.Bender@physik.lmu.de

**e-mail: Thomas.Kuhr@lmu.de

***e-mail: piilonen@vt.edu
}

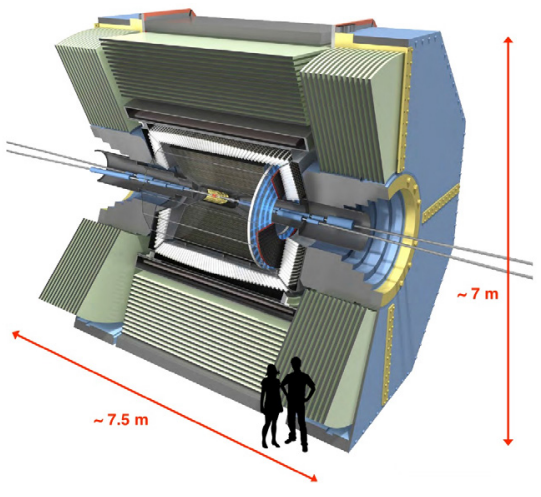

Figure 1: The Belle II detector [2]. 
electron-positron collision data. To visualize this data, amongst other things, Virtual Reality (VR) applications are used within the collaboration.

VR denotes a computer-generated scenario that simulates an immersive environment. VR became more and more popular in recent years, with the releases of the HTC Vive [3] and the Oculus Rift [4]. Both are so-called head-mounted displays which, through the combination of displays and lenses within the headset, give the user the impression of being in a virtual environment. A different approach to VR is done by so-called cave automatic virtual environments (CAVE) [5], where projectors are directed to up to six walls of a room-sized cube to generate the VR experience. Both approaches use tracking to capture the position and movements of the user in order to create an even more immersive experience. As example the HTC Vive with its tracking principle is shown in figure 2. To be used for education and outreach, Belle II has developed applications for both VR setups. Belle2VR, designed for HMDs, is described in section 2, while Belle II GRETCHEN, an application for CAVEs, is presented in section 3 .

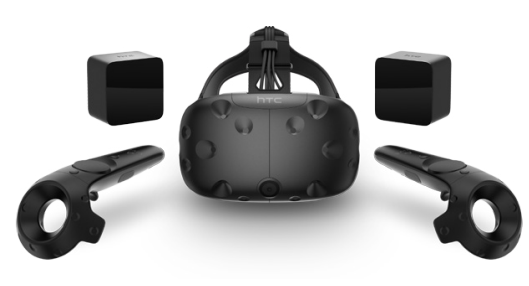

(a) HTC Vive

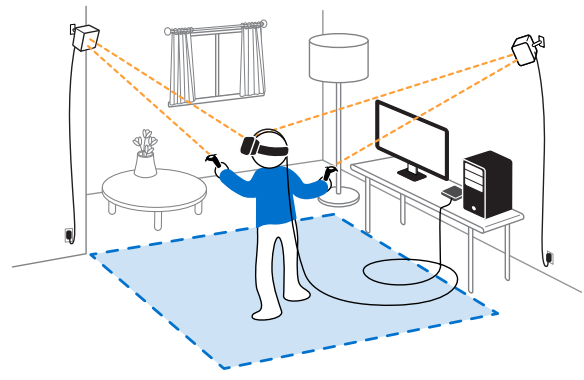

(b) Vive tracking

Figure 2: The HTC Vive, with its corresponding tracking mechanism [3]. The HMD and the controllers track their position with the help of so-called lighthouse boxes, which sweep the room with infrared and laser light.

\section{Belle2VR}

Belle2VR [6] has been developed as a pedagogical tool for high school and undergraduate students. It displays a 3D model of the Belle II detector together with simulated electronpositron collisions. Therefore Belle2VR allows users to investigate the detector and the evolution of the particle collisions itself. Belle2VR is developed with the Unity [7] game engine, which supports multiple operating systems, including VR and mobile platforms. Consequently Belle2VR is available for different platforms, like the HTC Vive, the Oculus Rift and the Oculus GO [8], in addition to a browser version, which uses WebGL [9]. The detector model used in the application is imported from the Geant4 [10] detector description incorporated in the Belle II software framework [11]. The displayed events are created with the full Belle II event simulation and detector reconstruction and therefore model the measured collisions in the experiment as realistically as possible. This information is saved as a .csv file and is subsequently imported into Unity. Since simulated data is used, the full event information is accessible, which otherwise would not be available to the user, e.g. the underlying physics process and the correct particle properties, like type, energy and momentum. The event dis- 
play is not static, which means that the event is experienced in 'real time' ${ }^{1}$. In order to explore the event, Belle2VR allows the user to move around freely within the virtual environment, but also the detector position, orientation and scale can be varied. In addition, users can toggle the visibility of detector components, investigate particle properties and control the event time. The purpose of Belle2VR is to introduce users to the concepts of particle physics but it also gives them the opportunity to explore features specific to the Belle II experiment. For example, students can rediscover the law of energy conservation in particle decays measured by the Belle II detector. An impression of Belle2VR is given in figure 3. Despite being highly

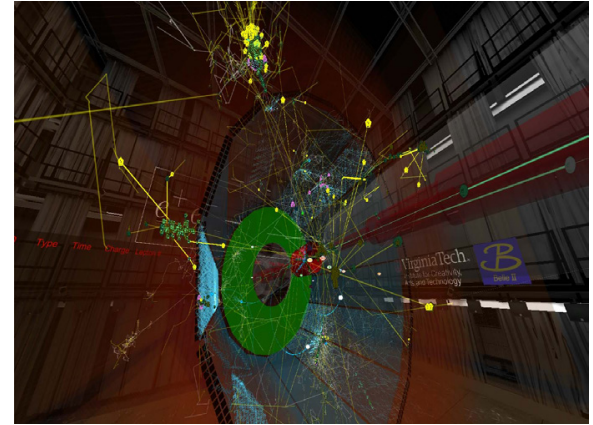

(a) Screenshot of Belle2VR

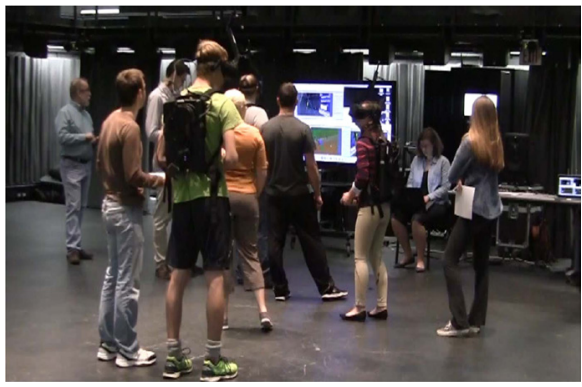

(b) Students using Belle2VR

Figure 3: Impressions of the Belle2VR application. Shown are a screenshot of the application and a picture taken during testing with students and developers [6].

topical, most people have not yet had the chance to experience VR. This makes VR a perfect tool to draw public attention to the Belle II experiment. Therefore a simplified version of Belle2VR has been developed [12], specifically designed for outreach events. The target audience for this application are people without any prior knowledge of (particle) physics, but who have a certain interest in the field. With the adapted version they can 'play' around with the application to get a feeling of what happens in a particle collision, without having to know anything about particle physics at all. Usually, during its use physics related questions arise, which can then be addressed right away, with the help of the VR event representation seen by the user. If the user is interested, this can serve as a starting point for a deepened conversation about the experiment or (particle) physics in general.

\section{Belle II GRETCHEN}

Besides head-mounted displays, there are also other technical approaches to VR. One of these alternatives is the cave automatic virtual environment (CAVE). A CAVE generates an immersive VR environment by illuminating the faces of a room-sized cube. To create the impression of stereoscopic depth, similar to the head-mounted displays, for each frame two images are rendered, one for the left and for the right eye. To ensure that only the eye for which the image has been rendered receives a picture, so-called shutter glasses are used, which alternately block the vision for one eye. The main advantage of the CAVE is, that it offers a deepened immersive perception. In contrast to head-mounted displays, where due to the technical approach, the user is completely locked out from its environment, in CAVE

\footnotetext{
${ }^{1}$ The typical timescale for an event is on the order of nanoseconds, therefore 'real time' means that the time interval is stretched to an observable timescale. However, users are free to adjust the timescale to their needs.
} 


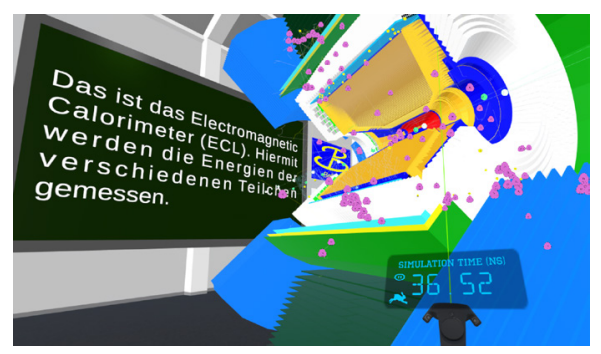

(a) Belle2VR screenshot

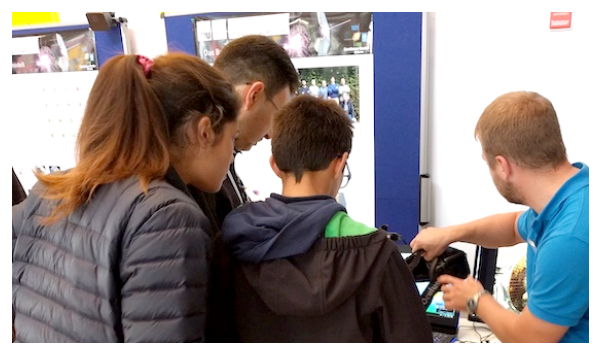

(b) Usage in outreach

Figure 4: Impressions of the adapted Belle2VR application. Shown are a screenshot of the application and a picture taken during a local outreach event [12].

installtations, users can still see their surroundings and also themeselves, due to the use of shutter glasses. This is especially valueable if multiple persons use the CAVE, since they can interact completely naturally within the VR environment. Such a Cave installation is available at the Leibniz Supercomputing Centre (LRZ) [13], for which a Belle II VR application, named graphical education tool for cave environments (GRETCHEN) [14], has been created.

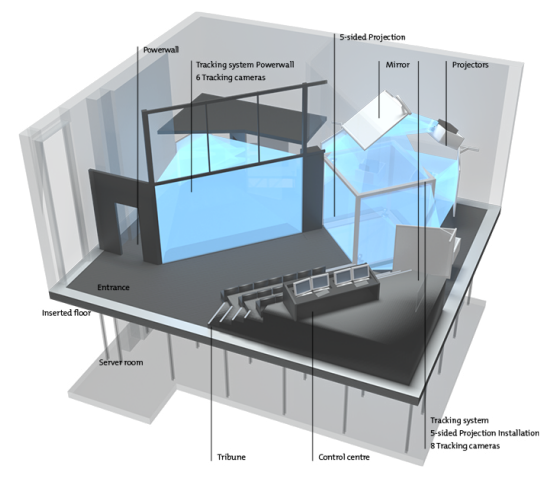

Figure 5: Layout of the Virtual Reality and Visualisation Centre at LRZ [13], the CAVE is located on the right.
The CAVE installation at LRZ, which is shown in figure 5 , consists of five $2.7 \mathrm{~m} \times 2.7 \mathrm{~m}$ faces, each illuminated with two $1080 \mathrm{p}$ projectors. It is driven by a cluster of twelve computing nodes, one render node for each projector and two nodes that control the other nodes and update the scene. The tracking is done by cameras, which register infrared light reflected by markers on the shutter glasses and controllers. Since Unity unfortunately does not support this CAVE installation, it was necessary to develop a new application completely from scratch, which is written in $\mathrm{C}++$ [15] and based on the OpenGL [16] application programming interface. GRETCHEN, as Belle2VR, is an interactive event display, which shows electronpositron collisions in the Belle II detector. It

follows the approach chosen by the outreach version of Belle2VR very closely and offers similiar features, like the control over the detector and event time. One key feature of GRETCHEN is the integration of a custom library which enables the CAVE support for this application. It is responsible for providing the tracking information, distributing the rendering tasks and synchronizing the nodes. Some impressions of the GRETCHEN application are shown in figure 6.

\section{Conclusion}

To visualize the electron-positron collisions ocuring in the Belle II detector, amongst other things, VR applications are used by the collaboration. As described there are two main applications at the moment, Belle2VR for head-mounted displays and GRETCHEN for CAVE 


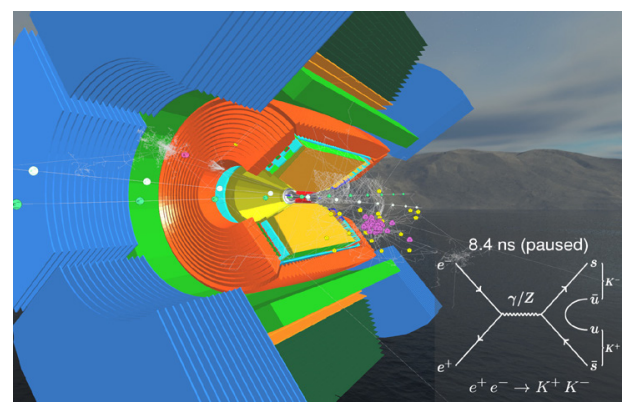

(a) Screenshot of GRETCHEN

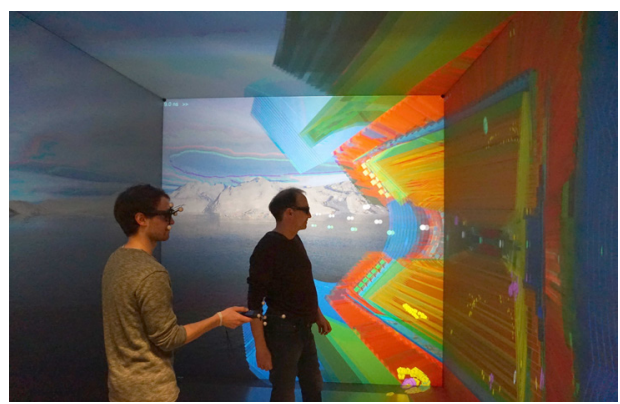

(b) Staff members using GRETCHEN

Figure 6: Impressions of GRETCHEN. Shown are a screenshot of the application and a picture taken during development [6].

environments. In addition to the nominal Belle2VR application, which is perfectly suited for high-school and undergraduate education, there is also a simplified version of the software, which is specifically designed for outreach activities. Since the only major experimentspecific parts of the Belle II VR applications are the detector model and the simulated events, those applications could easyly be modified for the use with other particle physics experiments.

\section{References}

[1] Abe T et al. 2010 Preprint, arXiv: 1011.0352

[2] Belle II experiment, www . belle2 . org

[3] HTC Vive is a product of HTC Corporation Ltd., www . vive. com

[4] Oculus Rift is a product of Oculus VR, LLC, www. oculus . com/rift

[5] Cruz-Neira C et al. 1992 Commun. ACM 35 64, doi : 10. 1145/129888. 129892

[6] Duer Z et al. 2018 IEEE Computer Graphics and Applications 38 33, doi : 10.1109/MCG. 2018.032421652

[7] Unity is a product of Unity Technologies SF, www . unity3d.com

[8] Oculus Go is a product of Oculus VR, LLC, www . oculus . com/go

[9] WebGL is managed by the Khronos Group, Inc., www . khronos.org/webgl

[10] Agostinelli S et al. 2003 Nucl. Instrum. Meth. A 506 250, doi : 10. 1016/S0168-9002(03)01368-8

[11] Kim D Y et al. 2017 J. Phys.: Conf. Ser. 898 042043, doi : $10.1088 / 1742-6596 / 898 / 4 / 042043$

[12] Adapted version of Belle2VR, www . flavor . physik.uni-muenchen.de

[13] VR and Visualisation Centre at the Leibniz Supercomputing Centre, www.lrz.de/services/v2c_en

[14] Belle II GRETCHEN, https://gitlab.lrz.de/Michael.Bender/BelleII-GRETCHEN

[15] $\mathrm{C}++$ is defined by the International Organization for Standardization, www . iso . org

[16] OpenGL is managed by the Khronos Group, Inc., www . opengl . org 